\title{
Noninvasive PET Imaging and Tracking of Engineered Human Muscle Precursor Cells for Skeletal Muscle Tissue Engineering
}

\author{
Deana Haralampieva ${ }^{1-3}$, Thomas Betzel ${ }^{1}$, Ivana Dinulovic ${ }^{4}$, Souzan Salemi² ${ }^{2}$ Meline Stoelting ${ }^{2}$, Stefanie D. Krämer ${ }^{1}$, \\ Roger Schibli ${ }^{1}$, Tullio Sulser ${ }^{2}$, Christoph Handschin ${ }^{4}$, Daniel Eberlii ${ }^{2,3}$, and Simon M. Ametamey ${ }^{1,3}$ \\ ${ }^{1}$ Institute of Pharmaceutical Sciences, ETH Zurich, Zurich, Switzerland; ${ }^{2}$ Laboratory for Tissue Engineering and Stem Cell Therapy, \\ Department of Urology, University Hospital Zurich, University of Zurich, Zurich, Switzerland; ${ }^{3}$ Zurich Center for Integrative Human \\ Physiology, Zurich, Switzerland; and ${ }^{4}$ Focal Area of Growth and Development, Biozentrum, University of Basel, Basel, Switzerland
}

Transplantation of human muscle precursor cells (hMPCs) is envisioned for the treatment of various muscle diseases. However, a feasible noninvasive tool to monitor cell survival, migration, and integration into the host tissue is still missing. Methods: In this study, we designed an adenoviral delivery system to genetically modify hMPCs to express a signaling-deficient form of human dopamine $\mathrm{D}_{2}$ receptor (hD2R). The gene expression levels of the receptor were evaluated by reverse transcriptase polymerase chain reaction, and infection efficiency was evaluated by fluorescent microscopy. The viability, proliferation, and differentiation capacity of the transduced cells, as well as their myogenic phenotype, were determined by flow cytometry analysis and fluorescent microscopy. ${ }^{18} \mathrm{~F}$-fallypride and ${ }^{18} \mathrm{~F}$-fluoromisonidazole, two well-established PET radioligands, were assessed for their potential to image engineered hMPCs in a mouse model and their uptakes were evaluated at different time points after cell inoculation in vivo. Biodistribution studies, autoradiography, and PET experiments were performed to determine the extent of signal specificity. To address feasibility for tracking hMPCs in an in vivo model, the safety of the adenoviral gene delivery was evaluated. Finally, the harvested tissues were histologically examined to determine whether survival of the transplanted cells was sustained at different time points. Results: Adenoviral gene delivery was shown to be safe, with no detrimental effects on the primary human cells. The viability, proliferation, and differentiation capacity of the transduced cells were confirmed, and flow cytometry analysis and fluorescent microscopy showed that their myogenic phenotype was sustained. ${ }^{18} \mathrm{~F}$-fallypride and ${ }^{18} \mathrm{~F}$ fluoromisonidazole were successfully synthesized. Specific binding of ${ }^{18} \mathrm{~F}$-fallypride to $\mathrm{hD} 2 \mathrm{R}$ hMPCs was demonstrated in vitro and in vivo. Furthermore, the ${ }^{18} \mathrm{~F}$-fluoromisonidazole signal was high at the early stages. Finally, sustained survival of the transplanted cells at different time points was confirmed histologically, with formation of muscle tissue at the site of injection. Conclusion: Our proposed use of a signaling-deficient $h D 2 R$ as a potent reporter for in vivo hMPC PET tracking by ${ }^{18} \mathrm{~F}$-fallypride is a significant step toward potential noninvasive tracking of hD2R hMPCs and bioengineered muscle tissues in the clinic.

\footnotetext{
Received Dec. 2, 2015; revision accepted Feb. 8, 2016.

For correspondence or reprints contact: Simon M. Ametamey, Institute of Pharmaceutical Sciences, ETH Zurich, Vladimir-Prelog-Weg 4, 8093-Zurich, Switzerland.

E-mail: simon.ametamey@pharma.ethz.ch

Published online May 19, 2016.

COPYRIGHT (C 2016 by the Society of Nuclear Medicine and Molecular Imaging, Inc.
}

Key Words: human muscle precursor cells; dopamine 2 receptor; hypoxia; in vivo imaging; PET

J Nucl Med 2016; 57:1467-1473

DOI: 10.2967/jnumed.115.170548

$\mathbf{O}$ rgan transplantation is currently the gold standard for rescuing damaged tissues but has several drawbacks such as dependence on donor organs and the high morbidity of immunosuppressive therapy. Regenerative medicine using autologous stem cells for organ and tissue replacement may offer an alternative that overcomes the known pitfalls (1-3). Tissue engineering, a major component of regenerative medicine, follows the principles of cell transplantation, materials science, and engineering toward development of biologic substitutes that can restore and maintain normal function (4). Because of their regenerative capacity, muscle precursor cells are investigated for skeletal muscle tissue reconstruction and replacement (5). These adult stem cells reside on the periphery of muscle fibers and are activated after injury, proliferating, differentiating into myoblasts, and later fusing to form new myofibers, thereby granting sufficient progeny for repetitive tissue repair (6). Most muscle precursor cells are committed to the myogenic lineage and are therefore the most suitable source for muscle engineering (7). Recent preclinical studies have shown that muscle reconstruction using muscle precursor cells is a promising and feasible therapy (8); however, the fate of the cells after implantation needs to be further investigated.

Currently, these issues are addressed by histologic assessment, which has one major shortcoming: the invasiveness of biopsies and the destruction of bioengineered muscle tissue. Novel noninvasive imaging technologies are therefore needed.

Molecular imaging is an emerging field providing essential information about heterogeneous human disorders. While bioluminescence has poor spatial resolution and MRI lacks the high sensitivity of radionuclide-based tools, PET/CT is a system with both high resolution and high sensitivity (9). Imaging reporter genes are available for fluorescence imaging, bioluminescence imaging, and MRI, but only radionuclide-based reporter genes are currently being investigated for use in patients (10-12). One such system is based on dopamine $\mathrm{D}_{2}$ receptor imaging using PET. Natively, expression of the dopamine $D_{2}$ receptor 
is limited largely to the striata nigra (13). A large number of specific and high-affinity dopamine $\mathrm{D}_{2}$ receptor PET ligands are available, some of which have found routine application in the clinic (14). Thus, PET imaging of exogenously added human dopamine $\mathrm{D}_{2}$ receptor $(\mathrm{hD} 2 \mathrm{R})$ in cells injected into peripheral body regions would be an attractive method to track $h D 2 R$ in vivo. One potential shortcoming of this approach, however, is the possible induction of undesired biologic effects by gene introduction and by alteration of intracellular signaling pathways. As binding of the dopamine $\mathrm{D}_{2}$ receptor ligand would activate the G-protein-linked signaling pathway, a mutated rat receptor (D2R80A) has been reported that uncouples ligand binding from intracellular signal transduction $(15,16)$. However, the amount of signal production is limited, as one receptor interacts with only one ligand molecule and can potentially be blocked by nonlabeled endogenous ligands (17).

Skeletal muscle cells are a key target for many stem cell and gene therapy applications. With PET imaging gaining increasing importance in regenerative medicine, an area of keen interest is the potential use of transfected muscle precursor cells to restore muscle function. In this study, our main aim was to investigate the location, level of expression, and survival duration of engineered human muscle precursor cells (hMPCs) using $\mathrm{hD} 2 \mathrm{R}$ as a reporter gene to determine the possibility of applying PET/CT imaging to noninvasively monitor implanted and genetically modified $\mathrm{hD} 2 \mathrm{R}$ hMPCs in a mouse model. The accomplishment of this goal would help to improve our understanding of the behavior of hMPCs during in vivo differentiation.

\section{MATERIALS AND METHODS}

\section{Isolation and Expansion of hMPCs}

Human biopsy samples from the musculus rectus abdominis were randomly collected after approval by the local institutional review board and after written informed consent had been obtained from hospitalized patients undergoing abdominal surgery. All samples were processed according to established protocols (18).

\section{Adenoviral Design}

The AdEasy system (Stratagene) (19) was used for recombinant adenovirus construction. Briefly, we mutated the phenylalanine-411 of $\mathrm{hD} 2 \mathrm{R}$ into alanine (F411A) to obtain a signaling-deficient $\mathrm{hD} 2 \mathrm{R}$ that still binds ligands in a normal manner but will not activate intracellular signaling on ligand binding (20). In detail, IRAUp969E0451D vector containing hD2R (ImaGenes) and pcDNA3 plasmid containing monomer red fluorescent protein (Addgene) were purchased. The $\mathrm{hD} 2 \mathrm{R}$ was subcloned into the pcDNA3.1 TOPO expressional vector (Invitrogen), which resulted in addition of C-terminal hexahistidine and V5 tags. Next, the F411A point mutation was introduced by site-directed mutagenesis (Stratagene). The $\mathrm{hD} 2 \mathrm{R}$ and monomer red fluorescent protein sequences were then subcloned into the backbone of the pShuttle cytomegalovirus vector (Addgene), ensuring a robust, constitutive expression. The final vector thus contained $\mathrm{hD} 2 \mathrm{R}$ and red fluorescent protein sequences, both running under two separate cytomegalovirus promoters. Successful cloning was validated by sequencing, whereas viral infection and gene expression were monitored by visualizing red fluorescent protein and reverse transcriptase polymerase chain reaction, respectively. The transduced cells were expanded for $2 \mathrm{~d}$ after infection and were subcutaneously injected into nude mice.

\section{Fiber Formation Assay}

Differentiation of hMPCs into myofibers in vitro was assayed as previously reported (22). The fusion rate was calculated by dividing the number of nuclei per myofiber by the number of nuclei per high-power field (\%). The imaging software ImageJ for Microscopy was used for data assessment.

\section{Cell Characterization}

After the expansion of hMPCs to passage 3, they were infected with the designed $\mathrm{hD} 2 \mathrm{R}$ adenovirus and cultured for $2 \mathrm{~d}$. The cells were characterized by fluorescence-activated cell sorting, using the following primary antibodies: anti-Pax7 (1:100; Sigma), anti-MyoD (1:100; BD), anti-myosin heavy-chain antibody (1:1; DSHB), antidesmin (1:50; Sigma), anti-sarcomeric $\alpha$-actinin (1:200; Sigma), anti-CD34 (1:100; $\mathrm{BD})$, and anti-IgG isotype control (1:100; Santa Cruise). Fluorescein isothiocyanate antimouse $\operatorname{IgM} \operatorname{IgG}$ polyclonal antibody (1:200; BD) was used as a secondary antibody. In total, 50,000 events were registered by fluorescence-activated cell sorting FACSCanto flow cytometry (BD Biosciences) and analyzed using FlowJo software, version 7.5 (Tree Star Inc.). All data are expressed as the percentage of maximum.

Additionally, the transduced hMPCs were cultured to $70 \%-80 \%$ confluency and stained with antidesmin (1:50), anti-sarcomeric $\alpha$-actinin (1:200), and anti-CD34 (negative control) (1:100). After the cells had been washed with phosphate-buffered saline, they were incubated with fluorescein isothiocyanate antimouse $\operatorname{IgG}$ IgM secondary antibody (1:500) or phalloidin (1:200; Sigma) and 4',6-diamidino2-phenylindole (1:100; Sigma) and finally mounted (Dako). Images were acquired with a model DM6000 B microscope (Leica) at exposures normalized to unstained controls (secondary antibody and 4',6diamidino-2-phenylindole only).

\section{Cell Viability and Proliferation}

In all cases, cell numbers and viability were confirmed by trypan blue staining. To evaluate the proliferation and viability of the infected cells at different time points, hMPCs were cultured for $6 \mathrm{~d}$. The cell proliferation reagent WST-1 (Roche) was used according to the manufacturer's protocol. To further confirm cell viability, hMPCs were stained with $10 \mu \mathrm{M}$ CellTrace calcein green acetoxymethyl (Life Technologies) for $30 \mathrm{~min}$ at $37^{\circ} \mathrm{C}$. Viable cells were detected using a fluorescence microscope. All measurements were repeated at least 3 times, and samples were analyzed in triplicate.

\section{Radiosynthesis of ${ }^{18} \mathrm{~F}$-Fallypride}

${ }^{18} \mathrm{~F}$-fallypride was synthesized in a 1-step reaction according to a published procedure (23). The radiotracer was obtained in a radiochemical purity of more than $95 \%$, and specific activity ranged from 32 to $168 \mathrm{GBq} / \mu \mathrm{mol}$.

\section{Radiosynthesis of ${ }^{18} \mathrm{~F}$-Fluoromisonidazole}

${ }^{18} \mathrm{~F}$-fluoromisonidazole was obtained in a 2-step reaction in analogy to a previously published method (24). The final formulated product was obtained in a decay-corrected radiochemical yield ranging from $25 \%$ to $37 \%$. Radiochemical purity was more than $95 \%$, and specific activities ranged from 34 to $190 \mathrm{GBq} / \mu \mathrm{mol}$.

\section{In Vitro ${ }^{18}$ F-Fallypride Uptake Assay}

The experiment was performed in longitudinally truncated 24-well plates testing $2 \mathrm{AV}-\mathrm{hD} 2 \mathrm{R}$ constructs in 3 concentrations $(500 \mathrm{ng}, 1 \mu \mathrm{g}$, $2 \mu \mathrm{g} /$ well). Approximately 20,000 hMPCs per well were seeded, and after $24 \mathrm{~h}$ the cells were infected with the corresponding constructs for $2 \mathrm{~d}$. The modified cells were incubated with ${ }^{18} \mathrm{~F}$-fallypride $(1 \mathrm{nM})$ for $15 \mathrm{~min}$ and scanned $26 \mathrm{~min}$ after the start of incubation. The cells were imaged using a small-animal PET scanner. The scan duration was $45 \mathrm{~min}$. All experiments were performed in triplicate.

\section{Animal Experimentation}

All animal experiments were approved by the local animal care committee. The study used a total of 46 female 8 -wk-old nude mice (Charles River). The hMPCs were expanded to passage 3-4 for the in 
vivo experiments. Each sample contained $30 \times 10^{6}$ transduced hMPCs, which were gently mixed with $500 \mu \mathrm{L}$ of collagen type I carrier (final concentration, $2 \mathrm{mg} / \mathrm{mL}$ ) (BD) and prepared for injection (25). Each animal received 2 subcutaneous injections, one on either side of the back (including control collagen-only injections). The engineered skeletal muscle tissues were harvested after 1, 2, and $4 \mathrm{wk}$.

\section{Tracking of Bioengineered Muscle Tissues via PET/CT Imaging}

PET experiments were performed with a dedicated small-animal PET/ CT tomograph (eXplore VISTA; General Electric), which uses phoswich detector technology in a dual-ring setup (26). For ${ }^{18} \mathrm{~F}$-fallypride administration, the mice were restrained and injected via a lateral tail vein with 4.38-26.25 MBq (0.17-2.34 nmol) of the radioligand. For blockage experiments, haloperidol $(1 \mathrm{mg} / \mathrm{kg})$ was injected. Ten minutes after radiotracer injection, the animals were anesthetized with isoflurane in an air-oxygen mixture and were positioned and fixed on the bed of the camera. PET data were acquired to yield dynamic images with sufficient count statistics. Depth of anesthesia and body temperature were monitored and controlled according to a previously published protocol (27). In addition, respiratory frequency was controlled with a model $1025 \mathrm{~T}$ monitoring system from SA Instruments. After the PET scan, the animals were allowed to recover from the anesthesia and were rescanned at a later time with the next tracer. Raw data were reconstructed by 2-dimensional ordered-subsets expectation maximization, and the obtained image data were evaluated by visual inspection and semiquantitative volume-of-interest analysis (28) using the dedicated software PMOD (PMOD Technologies Ltd.).

\section{Biodistribution Studies}

The subsequent postmortem biodistribution was assessed $60 \mathrm{~min}$ after intravenous injection of ${ }^{18} \mathrm{~F}$-fallypride. Tracer uptake in engineered muscle tissue, striatum nigra, native muscle tissue, cerebellum, and injected collagen was evaluated in a $\gamma$-counter (Cobra II AutoGamma; Canberra Packard).

\section{Autoradiography}

Cryosections $(20 \mu \mathrm{m})$ of samples were preincubated on ice for $12 \mathrm{~min}$ in Tris/ $\mathrm{HCl}, 0.1 \%$ bovine serum albumin, $\mathrm{pH}$ 7.4. Then, the slides were incubated with ${ }^{18} \mathrm{~F}$-fallypride $(0.03 \mathrm{nM})$ in the presence and absence of haloperidol $(10 \mu \mathrm{M})$ as a blocker for $60 \mathrm{~min}$. After the tissue slices had been air-dried, they were exposed to phosphor imager plates, which were analyzed with a BAS-5000 phosphor imager (Fuji).

\section{Histologic Assessment}

The harvested engineered muscle tissues were embedded in a cryopreservative optimal-cutting-temperature medium (CellPath) immediately after isolation. Cryosections were prepared $(10 \mu \mathrm{m})$ and further processed. Hematoxylin and eosin staining (Sigma) was performed according to the manufacturer's protocol.

\section{Reverse Transcriptase Polymerase Chain Reaction}

The infected cells were cultured for $2 \mathrm{~d}$ and harvested for analysis of $\mathrm{hD} 2 \mathrm{R}$ gene expression. Total RNA was isolated using the SV Total RNA Isolation System (Promega) according to the manufacturer's protocol. RNA was reverse-transcribed with random primers (highcapacity complementary DNA reverse transcription kit; Life Technologies). Predesigned primers for hD2R (Hs00241436_m1) and human vascular endothelial growth factor A (Hs00900055_m1) were purchased from Life Technologies. 18S ribosomal RNA (4319413E; Life Technologies) was used to normalize complementary DNA concentrations. For quantification, the expression of each gene was normalized to the $18 \mathrm{~S}$ expression in the corresponding sample. The entire experiment was repeated at least three times, and samples were analyzed in triplicate.

\section{Gel Electrophoresis and Immunoblotting}

Total protein was measured with a bicinchoninic acid protein assay kit (Thermo Scientific), and protein lysate $(30-50 \mu \mathrm{g})$ was loaded on a $10 \%$ or $12 \%$ gel (Bio-Rad). The primary antibodies used were anti-myosin heavy-chain (1:5, DSHB), anti-sarcomeric $\alpha$-actinin (1:2,000; Sigma), anti-Von Willebrand factor (1:500; Sigma), anti-hD2R (1:200; Santa Cruz), and anti-glyceraldehyde 3-phosphate dehydrogenase (1:2,500; Sigma). Finally, the membranes were washed and incubated with the appropriate horseradish peroxidase-conjugated secondary antibody (1:500; Amersham Pharmacia Biotech) for $1 \mathrm{~h}$. The signals were detected by the enhanced chemiluminescence method (Amersham). Data were analyzed by Image Studio Lite (Li-Cor) software and were represented as protein expression relative to glyceraldehyde 3-phosphate dehydrogenase.

\section{Statistics}

For statistical analysis, SPSS, version 22.0 (IBM), was used and graphs were drawn with Prism, version 5.04 (GraphPad Software, Inc.). All data were analyzed by Student $t$ tests or 1-way ANOVA, with Bonferroni or least-significant-difference post hoc analysis $(P<$ 0.05 was considered significant). All presented data are expressed as mean with corresponding SEM.

\section{RESULTS}

\section{Viability, Proliferation, and Differentiation Rates of Genetically Modified hD2R hMPCs}

The adenoviral construct containing hD2R (F411A) was generated and amplified for further use in hMPCs. Nontoxicity of the viral infection was visualized by a cell viability assay (calcein) (Fig. 1A) in which no significant differences between wild-type (WT) and hD2R-infected cells were detected. The transduction efficiency of approximately $25 \%$ infected cells was confirmed by fluorescent imaging, as the designed construct contained a red fluorophore, expressed under a separate cytomegalovirus promoter. This construct allowed assessment of cell transduction efficiency before transplantation. Using a fiber formation assay (29), we evaluated the ability of hMPCs to form myofibers in vitro. Comparison between WT and hD2R-overexpressing cells (Supplemental Fig. 1; supplemental materials are available at http://jnm. snmjournals.org) did not reveal any significant difference in number of nuclei per fiber $(P=0.8629)$ (WT: $8.325 \pm 0.6818, n=83$; hD2R: $8.184 \pm 0.4635, n=87)$, number of nuclei per high-power field (WT: $230.6 \pm 14.34, n=10$; hD2R: $216.9 \pm 10.71, n=10$ $[P=0.4539]$ ), or number of fibers per high-power field (WT: $8.3 \pm$ 0.8699, $n=10$; hD2R: $8.4 \pm 0.5812, n=10[P=0.9249])$. The calculated overall fusion did not differ between infected and WT cells either (WT: $30.95 \pm 3.289, n=10$; hD2R: $32.40 \pm 3.085$, $n=10[P=0.7512])$. Successful transduction was also confirmed by hD2R gene expression levels (Fig. 1B) $(n=3 \times 3, P=$ $0.005)$. Furthermore, the proliferation capacity of the WT and hD2R-overexpressing hMPCs was determined by cell proliferation assay over $3 \mathrm{~d}$ of culturing after transduction, showing no significant variations between the different groups (Fig. 1C) (WT: $2.2173 \pm 0.364, n=6$; hD2R: $2.0843 \pm 0314, n=6$ $[P=0.787$ at $3 \mathrm{~d}])$.

\section{Flow Cytometry and Immunocytochemistry Analysis of hD2R hMPCs}

The cells were analyzed by flow cytometry (Fig. 2A) initially and in parallel by immunocytochemistry (Fig. 2B) $2 \mathrm{~d}$ after infection, revealing a sustained muscle phenotype without significant alterations between the groups. 


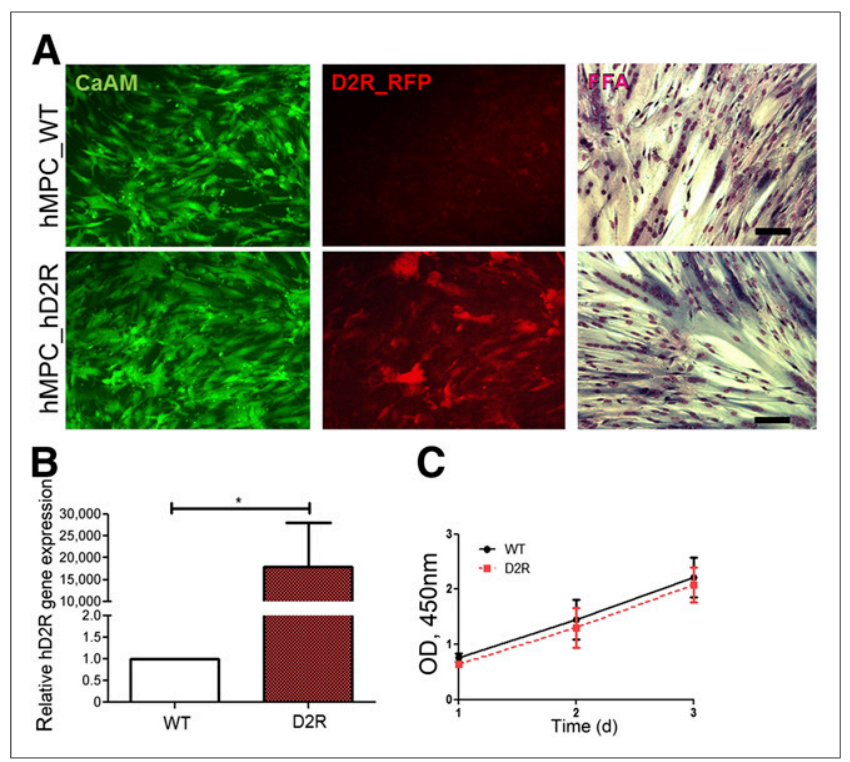

FIGURE 1. Evidence that $\mathrm{hD} 2 \mathrm{R}$ adenoviral infection did not show adverse effects on hMPC. (A) Adenoviral transduction of hMPCs with hD2R did not affect cell viability (calcein assay, left, green). Transduction efficiency was shown by fluorescence microscopy (middle, red). Ability to form fibers (29) was also not affected by virus (right). (B) hD2R gene expression was evaluated. (C) Proliferation rate did not differ between WT noninfected and infected hMPCs. All measurements were performed in duplicate from at least 3 different biopsies. ${ }^{\star} P<0.05$, Student $t$ test. CaAM $=$ calcein acetoxymethyl; FFA $=$ fiber-formation assay; $\mathrm{OD}=$ optical density; RFP = red fluorescent protein.

\section{In Vitro PET/CT Imaging of hD2R hMPCs Using ${ }^{18} \mathrm{~F}$-Fallypride}

Before each experiment, the infection efficiency was assessed by fluorescent microscopy, and positive cells were further processed for in vitro or in vivo studies. Initially, the capacity of the transduced hD2R hMPCs to efficiently bind ${ }^{18} \mathrm{~F}$-fallypride was evaluated in vitro. When using the designed adenovirus, testing 3 different concentrations of viral particles for tracer uptake, we observed a high binding affinity (Fig. 3A). As expected, the cells infected with the highest nontoxic concentration
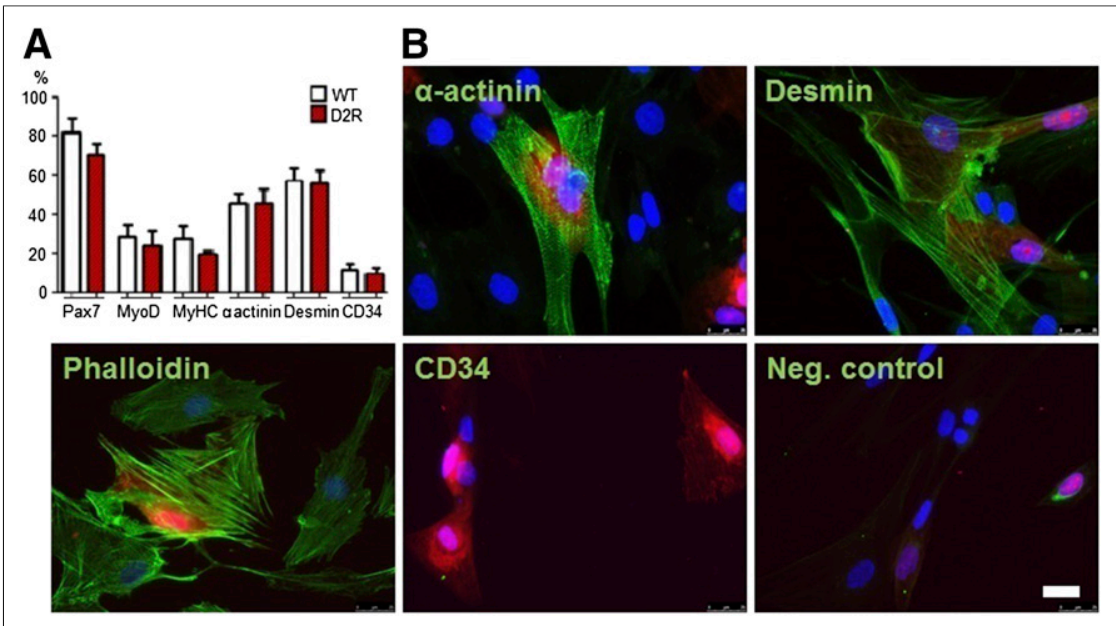

FIGURE 2. Evidence that adenoviral infection with hD2R did not change well-defined hMPC phenotype. (A) hMPCs were analyzed by flow cytometry. (B) Expression of typical muscle markers (green) in cells expressing hD2R (red) was further confirmed by immunocytochemistry. Nuclei are shown in blue. ${ }^{\star} P<0.05$, Student $t$ test $(n=4$ biopsies). Scale bar $=25 \mu \mathrm{m}$. $(\times 10)$ showed the highest detectable PET signal and were thus used for further experiments. These data confirmed the efficiency of the presented $\mathrm{hD} 2 \mathrm{R}$ adenoviral construct for hMPC genetic modification.

\section{In Vivo PET/CT Imaging of hD2R hMPCs Using ${ }^{18} \mathrm{~F}$-Fallypride}

Encouraged by our in vitro results, we evaluated the capability of transduced hMPCs to specifically bind ${ }^{18} \mathrm{~F}$-fallypride in an established ex situ model for skeletal muscle tissue formation in vivo. The hD2R hMPCs showed a high SUV after $1 \mathrm{wk}(0.259 \pm$ $0.019, n=8)$. This SUV decreased after 2 wk $(0.169 \pm 0.019$, $n=8[P=0.005])$, and no significant difference from background was observed after $4 \mathrm{wk}(0.134 \pm 0.014, n=6[P<$ 0.0001]) (Fig. 3B). Animals injected with only collagen did not show a significant radiotracer accumulation at any time $(0.115 \pm$ $0.011, n=10)$. Cerebellum was used as a negative control, showing negligible nonspecific uptake $(0.124 \pm 0.006, n=16)$. This was confirmed by volume-of-interest analysis (28), by which a significantly higher signal-to-background ratio of at least 2.08 at the earliest time point was observed (Fig. 3B). Biodistribution assessment also showed a significant decrease in tracer uptake in engineered tissues over time (Fig. 4A, $n=4-$ 16 per time point). Signal specificity was demonstrated by in vivo PET studies using haloperidol as an $\mathrm{hD} 2 \mathrm{R}$-blocking agent; the striatum was visualized as a positive control (Fig. 4B). Additionally, radioligand binding specificity was demonstrated by autoradiography studies, whereby hD2R-expressing tissues (including native controls) showed a reduction of radioligand uptake after incubation with haloperidol (Fig. 4C), confirming the in vivo data.

\section{PET Imaging of Hypoxia Using ${ }^{18}$ F-Fluoromisonidazole During Formation of Bioengineered Skeletal Muscle Tissue}

After successful tracking of the injected $\mathrm{hD} 2 \mathrm{R}$ hMPCs, we were further interested in studying the oxygenation status of the cells after implantation. To address this, we used ${ }^{18}$ F-fluoromisonidazole, a PET radiotracer for visualizing hypoxic regions. One week after cell inoculation, we observed a relatively high ${ }^{18} \mathrm{~F}$-fluoromisonidazole uptake, which decreased at later time points (Fig. 5A). Volume-ofinterest analysis of the 1 -wk samples $(0.315 \pm 0.02, n=8)$ showed a mean signal change ratio of 1.6, compared with later time points $(0.189 \pm 0.019, n=8$ [2 wk]; $0.205 \pm 0.009, n=6[4 \mathrm{wk}])$ and negative controls (cerebellum: $0.206 \pm$ 0.017, $n=16$; collagen: $0.251 \pm 0.003$, $n=10$ ) (Fig. 5B).

\section{Histologic Assessment and Gene and Protein Analysis of Harvested Tissues}

Western blotting was used to evaluate the relative protein expression of typical skeletal muscle protein markers (sarcomeric $\alpha$-actinin, myosin heavy-chain), Von Willebrand factor, and $\mathrm{hD} 2 \mathrm{R}$ after tissue harvesting (Fig. 6A). The tibialis anterior and striatum nigra were used as controls. The protein expression levels of sarcomeric markers gradually increased in the bioengineered tissue samples (myosin heavy-chain: $0.4211 \pm 0.033, n=4$ [1 wk]; $2.1915 \pm 0.407, n=6[2 \mathrm{wk}]$; $3.0734 \pm 0.72, n=8[4 \mathrm{wk}][P=$ 0.012]; $\alpha$-actinin: $0.4065 \pm 0.111, n=4$ 


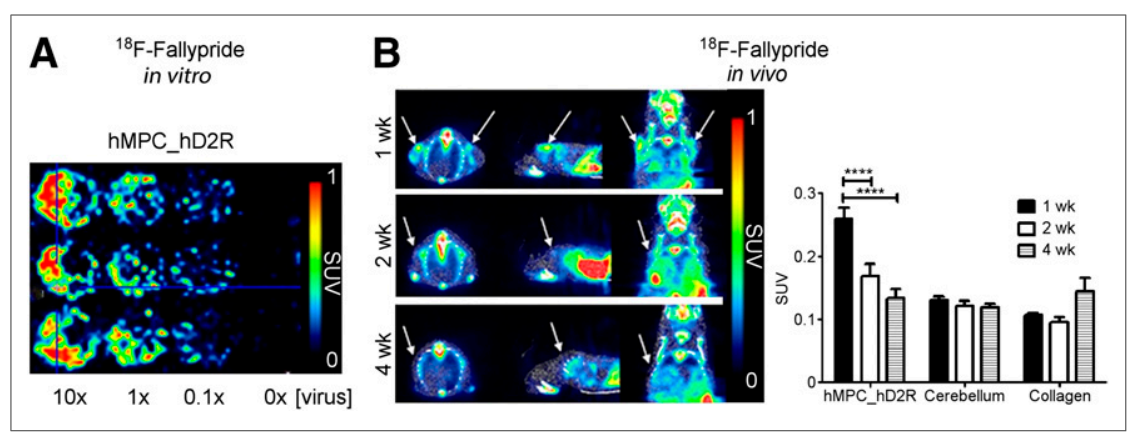

FIGURE 3. Tracking of hMPCs and engineered muscle tissue via PET/CT using ${ }^{18} \mathrm{~F}$-fallypride in vitro and in vivo. (A) ${ }^{18} \mathrm{~F}$-fallypride uptake in $\mathrm{hD} 2 \mathrm{R}$ hMPCs was detected at different adenoviral $\mathrm{hD} 2 \mathrm{R}$ transduction levels. (B) In vivo PET/CT imaging visualized specific tracer uptake in bioengineered tissues over time, and VOI analysis illustrated gradual decrease in signal after $1 \mathrm{wk}(n=$ $6-8)$. VOI = volume of interest.

[1 wk]; $0.6734 \pm 0.113, n=6[2 \mathrm{wk}] ; 1.2696 \pm 0.315, n=8$ [4 wk] $[P=0.043])$, corresponding to the expected timeline of muscle fiber formation (Fig. 6A). The relative $\mathrm{hD} 2 \mathrm{R}$ protein expression decreased steadily over time $(2.4072 \pm 0.608, n=3[1 \mathrm{wk}] ; 1.5881 \pm 0.25$, $n=6[2 \mathrm{wk}] ; 1.0394 \pm 0.294, n=12[4 \mathrm{wk}][P=0.031]$ ) (Fig. 6A), concomitant with the observed decrease in ${ }^{18} \mathrm{~F}$-fallypride uptake (Fig. 3B). The decrease in ${ }^{18} \mathrm{~F}$-fluoromisonidazole uptake over time coincided with an increase in Von Willebrand factor protein levels (endothelial cell marker, $n=4$ ) (Fig. 6A), as well as with rising expression of vascular endothelial growth factor-A gene (Supplemental Fig. 2B, $n=9$ ) in the bioengineered muscle tissues. Although $\mathrm{hD} 2 \mathrm{R}$ protein levels were decreasing over time, the corresponding gene expression levels were increasing (Supplemental Fig. 2B). The gradual decrease in size due to collagen remodeling and myofiber formation has previously been reported (25) (Supplemental Fig. 2A). Histologic analysis revealed successful myofiber formation in the

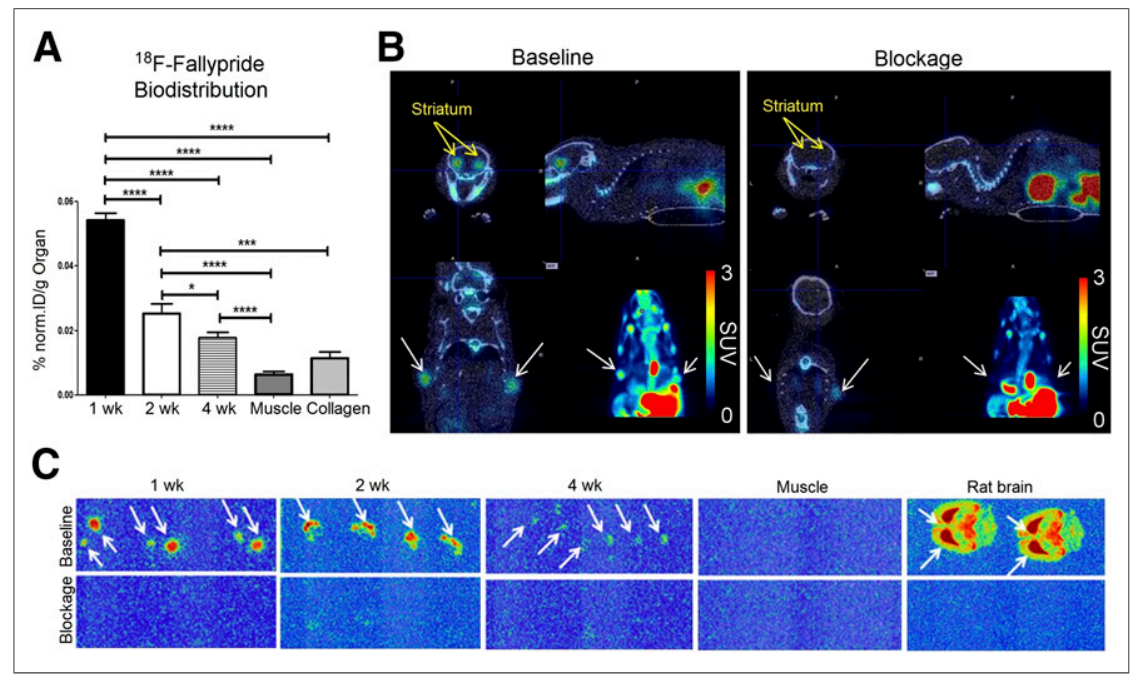

FIGURE 4. Specificity of ${ }^{18} \mathrm{~F}$-fallypride signal. (A) Significant enhancement of ${ }^{18} \mathrm{~F}$-fallypride uptake in engineered hD2R hMPC tissue, compared with collagen and control muscle, was observed in biodistribution study $(n=4-16)$. (B) Tracer specificity was evaluated after blockage experiment with haloperidol. (C) Autoradiography (top) showed specific tracer binding. Blockage (bottom) was performed with haloperidol, and mouse muscle and rat brain sections were used as negative and positive controls, respectively. Images were averaged from 60 to $120 \mathrm{~min}$. Arrows indicate hMPC injection site. ID = injected dose. ${ }^{*} P<0.05,1$-way ANOVA with Bonferroni post hoc analysis. harvested tissues and was able to illustrate the individual cells of the samples at week 1 and the myotube formation at weeks 2 and 4 (Fig. 6B).

\section{DISCUSSION}

Transplantation of muscle precursor cells has been contemplated as a treatment option for genetic and acquired muscle disorders $(25,30)$ and as a prospective way to reestablish damaged muscle function in patients with muscle degeneration (31). Various research groups have demonstrated the formation of muscle tissue after cellular inoculation (32). However, the long-term noninvasive tracking of bioengineered muscle tissue has not been addressed so far.

The need for a system combining well-established PET probes and a novel engineered vector for expression of $\mathrm{hD} 2 \mathrm{R}$ in hMPCs has prompted our research toward noninvasive visualization of a cell therapy for application in skeletal muscle bioengineering. The generation of a vector coding for a signaling-deficient $\mathrm{hD} 2 \mathrm{R}$ was an important milestone, as redundant cell-signaling is a major issue in gene therapies.

In this paper, we have described a method for metabolic imaging and tracking of hMPCs using PET/CT, thereby illustrating their exact position and the oxygenation state of the newly formed skeletal muscle tissue over time. Our study indicated that the signaling-deficient $\mathrm{hD} 2 \mathrm{R}$ could be efficiently introduced into primary hMPCs, that there were no detrimental effects on cell viability or differentiation, that myogenic marker gene expression was unaltered, that the receptor could be traced with high specificity using ${ }^{18} \mathrm{~F}$-fallypride both in cells in vitro and after injection in vivo, and that receptor expression in the engineered tissues could be validated by autoradiography. We found that the capacity to track $\mathrm{hD} 2 \mathrm{R}$ hMPCs is excellent in the first week and declines later. Interestingly, although the messenger RNA expression of $\mathrm{hD} 2 \mathrm{R}$ remained high, its protein levels steadily declined. This finding indicates that the ectopically expressed $\mathrm{hD} 2 \mathrm{R}$ is either posttranslationally modified or internalized on constitutive expression and ultimately degraded during the final differentiation to myofibers. Our data showed that the use of a high-affinity PET ligand and a mutated $\mathrm{hD} 2 \mathrm{R}$ can provide important information on localization, survival, and metabolic features in the early phase after cell injection.

A general problem in tissue engineering is the increased oxygen demand and lack of initial vascularization during the early phases of tissue formation, leading to central necrosis (33). Any engineered tissue larger than $0.3 \mathrm{~cm}^{3}$ requires rapid vascularization to guarantee survival of cells within the core of the construct. Vascularization of bioengineered tissue is therefore 


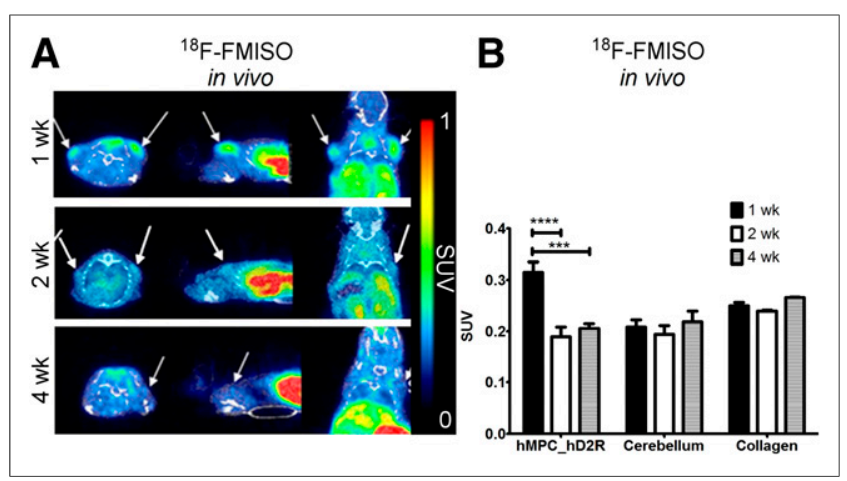

FIGURE 5. Hypoxia PET/CT monitoring with ${ }^{18} \mathrm{~F}$-fluoromisonidazole during formation of bioengineered muscle tissues. (A) Redox state of forming tissues was illustrated with ${ }^{18} \mathrm{~F}$-fluoromisonidazole (injected activity, 0.49-5.86 nmol, 14.86-35.83 MBq). (B) VOI signal was calculated for 3 different time points. Images were averaged from 90 to $110 \mathrm{~min}$. Arrows indicate hMPC injection sites.

a bottleneck. Tissue hypoxia, which is inversely proportional to the degree of vascularization, can be analyzed by invasive and noninvasive methods. The oxygenation status of cells and tissues can be determined by invasive $\mathrm{pO}_{2}$ measurements using polarographic needle electrodes or with fiber-optic probes (34-36). Noninvasive methods include the use of bioreductive chemical markers such as 2-nitroimidazoles (37), which form adducts under hypoxic conditions and are irreversibly bound in hypoxic cells. ${ }^{18} \mathrm{~F}$-fluoromisonidazole is the most widely used PET radioligand for imaging hypoxia in humans (38-40).

In our study with ${ }^{18} \mathrm{~F}$-fluoromisonidazole, we detected metabolically active bioengineered tissues with low oxygen levels, that is, tissues in which reductive reactions dominate because of a low oxygen supply or a high oxygen consumption, typical of the early stages of cell-to-myofiber formation. It has been shown that the level of oxygenation is influenced by the degree of vascularization and by the metabolic consumption of oxygen by engineered tissue (41). Concomitant with our in vivo observations, increased release of vascular endothelial growth factor in a hypoxic environment has been shown to lead to enhanced differentiation (42). Histologic analysis confirmed the cell-tomyofiber transition, correlating with normalized vascularization levels. In line with these observations, the use of ${ }^{18} \mathrm{~F}$ fluoromisonidazole for hypoxia imaging of bioengineered constructs seems feasible.

\section{CONCLUSION}

Knowledge of the fate of the inoculated primary hMPCs is a prerequisite for future clinical implementation of this cell therapy. Combining the recent developments in designing robust vectors along with novel, highly specific PET imaging probes, we were able to offer an elegant solution, obviating a destructive tissue biopsy. Our study marks a significant step toward the noninvasive tracking of hD2R hMPCs and bioengineered muscle tissues in the clinic using PET.

\section{DISCLOSURE}

The costs of publication of this article were defrayed in part by the payment of page charges. Therefore, and solely to indicate this fact, this article is hereby marked "advertisement" in accordance with 18 USC section 1734 . No potential conflict of interest relevant to this article was reported.

\section{ACKNOWLEDGMENTS}

We thank Damina Balmer for critical assessment of the manuscript and Claudia Keller for technical assistance with the $\mathrm{PET} / \mathrm{CT}$ scanner.

\section{REFERENCES}

1. Chun SY, Cho DH, Chae SY, et al. Human amniotic fluid stem cell-derived muscle progenitor cell therapy for stress urinary incontinence. J Korean Med Sci. 2012;27:1300-1307.

2. Nikolavasky D, Stangel-Wojcikiewicz K, Stec M, et al. Stem cell therapy: a future treatment of stress urinary incontinence. Semin Reprod Med. 2011;29:61-70.

3. Aref-Adib M, Lamb BW, Lee HB, et al. Stem cell therapy for stress urinary incontinence: a systematic review in human subjects. Arch Gynecol Obstet. 2013;288:1213-1221.

4. Olson JL, Atala A, Yoo JJ. Tissue engineering: current strategies and future directions. Chonnam Med J. 2011;47:1-13.

5. Smaldone MC, Chancellor MB. Muscle derived stem cell therapy for stress urinary incontinence. World $J$ Urol. 2008;26:327-332.

6. Hill M, Wernig A, Goldspink G. Muscle satellite (stem) cell activation during local tissue injury and repair. J Anat. 2003;203:89-99.

7. Benchaouir R, Rameau P, Decraene C, et al. Evidence for a resident subset of cells with SP phenotype in the C2C12 myogenic line: a tool to explore muscle stem cell biology. Exp Cell Res. 2004;294:254-268.

8. Eberli D, Aboushwareb T, Soker S, et al. Muscle precursor cells for the restoration of irreversibly damaged sphincter function. Cell Transplant. 2012;21:2089-2098.

9. Haralampieva DG, Ametamey SM, Sulser T, Eberli D. Non-invasive imaging modalities for clinical investigation in regenerative medicine. In: Eberli D, ed. Cells and Biomaterials in Regenerative Medicine. Rijeka, Croatia: InTech; 2014:175-197.

10. Peñuelas I, Mazzolini G, Boan JF, et al. Positron emission tomography imaging of adenoviral-mediated transgene expression in liver cancer patients. Gastroenterology. 2005;128:1787-1795. 
11. Peñuelas I, Haberkorn U, Yaghoubi S, et al. Gene therapy imaging in patients for oncological applications. Eur J Nucl Med Mol Imaging. 2005;32(suppl 2):S384-S403.

12. Dempsey MF, Wyper D, Owens J, et al. Assessment of ${ }^{123}$ I-FIAU imaging of herpes simplex viral gene expression in the treatment of glioma. Nucl Med Commun. 2006;27:611-617.

13. MacLaren DC, Gambhir SS, Satyamurthy N, et al. Repetitive, non-invasive imaging of the dopamine $\mathrm{D}_{2}$ receptor as a reporter gene in living animals. Gene Ther. 1999;6:785-791.

14. Siessmeier T, Zhou Y, Buchholz HG, et al. Parametric mapping of binding in human brain of $\mathrm{D}_{2}$ receptor ligands of different affinities. J Nucl Med. 2005;46:964-972.

15. Liang Q, Satyamurthy N, Barrio JR, et al. Noninvasive, quantitative imaging in living animals of a mutant dopamine $\mathrm{D}_{2}$ receptor reporter gene in which ligand binding is uncoupled from signal transduction. Gene Ther 2001;8: 1490-1498.

16. Schönitzer V, Haasters F, Kasbauer S, et al. In vivo mesenchymal stem cell tracking with PET using the dopamine type 2 receptor and ${ }^{18} \mathrm{~F}$-fallypride. $\mathrm{J} \mathrm{Nucl}$ Med. 2014;55:1342-1347.

17. Rodriguez-Porcel M. In vivo imaging and monitoring of transplanted stem cells: clinical applications. Curr Cardiol Rep. 2010;12:51-58.

18. Eberli D, Soker S, Atala A, et al. Optimization of human skeletal muscle precursor cell culture and myofiber formation in vitro. Methods. 2009;47:98-103.

19. Luo J, Deng ZL, Luo X, et al. A protocol for rapid generation of recombinant adenoviruses using the AdEasy system. Nat Protoc. 2007;2:1236-1247.

20. Cho W, Taylor LP, Mansour A, et al. Hydrophobic residues of the $\mathrm{D}_{2}$ dopamine receptor are important for binding and signal transduction. J Neurochem. 1995; 65:2105-2115.

21. Herzog H, Langen KJ, Weirich $\mathrm{C}$, et al. High resolution BrainPET combined with simultaneous MRI. Nuklearmedizin. 2011;50:74-82.

22. Eberli D, Soker S, Atala A, et al. Optimization of human skeletal muscle precursor cell culture and myofiber formation in vitro. Methods. 2009;47:98-103.

23. Mukherjee J, Yang ZY, Das MK, et al. Fluorinated benzamide neuroleptics-III. Development of (S)-N-[(1-allyl-2-pyrrolidinyl)methyl]-5-(3-[ $\left.{ }^{18} \mathrm{~F}\right]$ fluoropropyl)-2, 3-dimethoxybenzamide as an improved dopamine D-2 receptor tracer. Nucl Med Biol. 1995;22:283-296.

24. Lim JL, Berridge MS. An efficient radiosynthesis of $\left[{ }^{18} \mathrm{~F}\right]$ fluoromisonidazole. Appl Radiat Isot. 1993;44:1085-1091.

25. Delo DM, Eberli D, Williams JK, et al. Angiogenic gene modification of skeletal muscle cells to compensate for ageing-induced decline in bioengineered functional muscle tissue. BJU Int. 2008;102:878-884.

26. Wang Y, Seidel J, Tsui BM, et al. Performance evaluation of the GE Healthcare eXplore VISTA dual-ring small-animal PET scanner. J Nucl Med. 2006;47:1891-1900.
27. Honer M, Bruhlmeier M, Missimer J, et al. Dynamic imaging of striatal $\mathrm{D}_{2}$ receptors in mice using quad-HIDAC PET. J Nucl Med. 2004;45:464-470.

28. Dubé D, Brideau C, Deschenes D, et al. 2-heterosubstituted-3-(4-methylsulfonyl) phenyl-5-trifluoromethyl pyridines as selective and orally active cyclooxygenase-2 inhibitors. Bioorg Med Chem Lett. 1999;9:1715-1720.

29. Herzog H, Langen KJ, Weirich C, et al. High resolution BrainPET combined with simultaneous MRI. Nuklearmedizin. 2011;50:74-82.

30. Mitchell PO, Pavlath GK. Skeletal muscle atrophy leads to loss and dysfunction of muscle precursor cells. Am J Physiol Cell Physiol. 2004;287:C1753-C1762.

31. Stölting MN, Hefermehl LJ, Tremp M, et al. The role of donor age and gender in the success of human muscle precursor cell transplantation. J Tissue Eng Regen Med. July 23, 2014 [Epub ahead of print].

32. Putman CT, Sultan KR, Wassmer T, et al. Fiber-type transitions and satellite cell activation in low-frequency-stimulated muscles of young and aging rats. J Gerontol A Biol Sci Med Sci. 2001;56:B510-B519.

33. Lovett M, Lee K, Edwards A, et al. Vascularization strategies for tissue engineering. Tissue Eng Part B Rev. 2009;15:353-370.

34. Hockel M, Schlenger K, Aral B, et al. Association between tumor hypoxia and malignant progression in advanced cancer of the uterine cervix. Cancer Res. 1996;56:4509-4515.

35. Nordsmark M, Overgaard M, Overgaard J. Pretreatment oxygenation predicts radiation response in advanced squamous cell carcinoma of the head and neck. Radiother Oncol. 1996;41:31-39.

36. Nordsmark M, Alsner J, Keller J, et al. Hypoxia in human soft tissue sarcomas: adverse impact on survival and no association with p53 mutations. Br J Cancer. 2001;84:1070-1075.

37. Machulla H-J, ed. Imaging of Hypoxia: Tracer Developments. Dordrecht, The Netherlands: Kluwer Academic Publishers; 1999.

38. Bruehlmeier M, Roelcke U, Schubiger PA, et al. Assessment of hypoxia and perfusion in human brain tumors using PET with ${ }^{18} \mathrm{~F}$-fluoromisonidazole and ${ }^{15} \mathrm{O}_{-} \mathrm{H}_{2} \mathrm{O}$. J Nucl Med. 2004;45:1851-1859.

39. Kawai N, Lin W, Cao WD, et al. Correlation between ${ }^{18} \mathrm{~F}$-fluoromisonidazole PET and expression of HIF-1alpha and VEGF in newly diagnosed and recurrent malignant gliomas. Eur J Nucl Med Mol Imaging. 2014;41:1870-1878.

40. Muzi M, Peterson LM, O'Sullivan JN, et al. ${ }^{18}$ F-fluoromisonidazole quantification of hypoxia in human cancer patients using image-derived blood surrogate tissue reference regions. J Nucl Med. 2015;56:1223-1228.

41. Phelps EA, Garcia AJ. Update on therapeutic vascularization strategies. Regen Med. 2009;4:65-80.

42. Bryan BA, Walshe TE, Mitchell DC, et al. Coordinated vascular endothelial growth factor expression and signaling during skeletal myogenic differentiation. Mol Biol Cell. 2008;19:994-1006. 